\title{
Idiopathic Christmas tree cataract
}

\section{Una catarata idiopática en árbol de Navidad}

Diogo Lopes*

Department of Ophthalmology, Garcia de Orta Hospital, Almada, Portugal
A healthy 87-year-old female without relevant diseases came for consultation complaining of low vision over the past year, more noticeable in the left eye. Her best-corrected visual acuity (BCVA) measured by decimal scale was $8 / 10$ in OD and $4 / 10$ in OS. Slit-lamp examination revealed mild nuclear sclerosis in OD and hyper-reflective polychromatic needle-shaped deposits in the lens in OS, suggesting a diagnosis of Christmas tree cataract (Fig. 1). Usually, this type of cataract has no significant impact on VA. However, with a normal fundoscopic examination and the absence of other causes, the patient underwent phacoemulsification that achieved a significant improvement of BCVA to $9 / 10$ in OS.

\section{Conflicts of interest}

The authors declare that they do not have any conflicts of interest.

\section{Ethical disclosures}

Protection of human and animal subjects. The authors declare that the procedures followed were in accordance with the regulations of the relevant clinical research ethics committee and with those of the Code

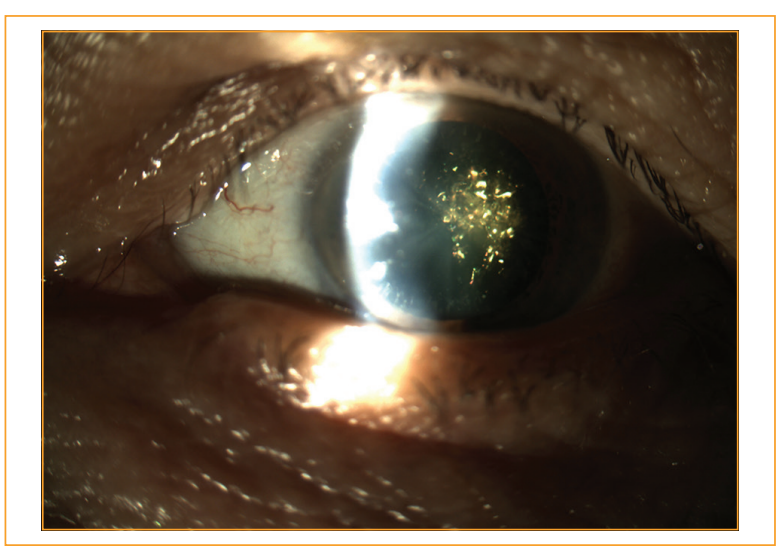

Figure 1. Direct focal illumination showed multiple hyper-reflective and polychromatic needle-shaped crystals in the left lens.

of Ethics of the World Medical Association (Declaration of Helsinki).

Confidentiality of data. The authors declare that they have followed the protocols of their work center on the publication of patient data.

Right to privacy and informed consent. The authors have obtained the written informed consent of the patients or subjects mentioned in the article. The corresponding author is in possession of this document. 